\title{
Will Canada have a rebound flu season this year?
}

- Cite as: CMAJ 2021 November 1;193:E1669-70. doi: 10.1503/cmaj.1095969

Posted on cmajnews.com on October 15, 2021

ockdowns and COVID-19 restrictions essentially quashed North America's flu season last year, but some experts worry there may be a rebound in viral infections this year as communities ease pandemic precautions.

Mask mandates, physical distancing and travel restrictions reduced the incidence of influenza in the United States by more than $60 \%$ last year, according to researchers from Columbia University's Mailman School of Public Health.

In a preprint article in The Journal of Infectious Diseases, researchers warned that health care systems need to prepare for severe influenza surges as COVID-19 measures relax.

Others worry that more people will be "immunologically naïve" and thus more vulnerable to the flu and other viral infections after low exposure last year. In another preprint study, researchers from the University of Pittsburgh predicted a "large compensatory influenza season" this year with $20 \%$ more hospitalizations than usual pre-COVID.

"Longer periods of decreased infection are followed by higher and earlier epidemic peaks, due to the increase in susceptibility in the population," they explained. "Higher influenza vaccine uptake is necessary to reduce the projected increase."

\section{Flu activity remains low for now}

In Canada, tens of thousands of flu cases would be considered normal in a typical season.

FluWatch, the national influenza surveillance system, reported more than 55000 flu cases in the 2019-20 season. By comparison, there were approximately 60 in 2020-21.

So far this year, influenza activity across Canada remains "exceptionally low," says Dawn Bowdish, Canada Research Chair in Aging and Immunity at McMaster University. "Of course, this could change."

Between August 29 and September 25, FluWatch reported just eight confirmed flu cases and no related hospitalizations. In a normal year, there may be 10 times as many cases over the same period.

"We don't know if that's because travel hasn't reached pre-pandemic levels. It could also be because in some of the most vulnerable populations where we usually see flu first - like in long-term care - there are still a lot of infection control measures in place," says Bowdish.

Canada often looks to the Southern Hemisphere as a harbinger of what to expect with our own influenza season. Both Australia and New Zealand have had "remarkably low influenza seasons," says Dr. Gerald Evans, chair of infectious diseases at Queen's University in Kingston, Ont.

However, "we have seen a surprising jump in influenza already in the Northern Hemisphere in India," Evans notes. "The situation arising in India does suggest the possibility that now that we're travelling more and now that public health measures have been, unfortunately, in some places reduced a bit, then we're likely to see more influenza than last year."

In a recent study in Influenza and Other Respiratory Viruses, Australian researchers write that even though influ- enza circulation was low in the Southern Hemisphere, it is "still present in a number of different countries and could pose a renewed threat in the upcoming Northern Hemisphere winter."

Other viruses resurging early in kids Meanwhile, it's unclear how last year's lockdowns and school closures will affect children's vulnerability to flu and other infections this year.

Children usually gain their first immunity against influenza from their mothers during pregnancy, or via vaccinations or infection in their first years of life. "By five years, you're exposed to a lot of things, [but during the pandemic] these kids will have missed almost two years of that exposure," says Bowdish.

Pediatric hospitals across Canada are already reporting resurgences of other respiratory viruses. Waves of children have been hospitalized for respiratory syncytial virus (RSV), a seasonal virus that typically emerges in the winter but has made an early comeback this year.

The United Kingdom has reported record numbers of hospitalizations for RSV in recent months, and Australia has seen more older children being affected than in previous years.

These unusual transmission patterns are an indirect consequence of COVID-19 lockdown measures, says Bowdish. "RSV is our example of how things could go very wrong [with influenza] if you protect a vulnerable population for a couple of years. Then you not only have the zero- to two-year-olds, but also the three- and four-year-olds getting sick because they missed out [on building immunity in their first years of life]." 
Dr. Mahli Brindamour, a pediatrician and assistant professor at the University of Saskatchewan, has noted "highly unusual" numbers of children hospitalized in Saskatoon with rhinovirus, adenovirus, non-COVID coronavirus and metapneumovirus since the summer months.

She worries that even a normal flu season combined with the fourth wave of COVID-19 and resurgences of other viruses could overwhelm health care systems, especially pediatric services. Adult patients with COVID-19 are already occupying pediatric intensive care beds in Saskatoon because of lack of capacity.

With more viruses circulating, there may also be a greater risk of people contracting multiple infections simultaneously. "We don't have a lot of data yet on the combination of COVID and RSV, or COVID and flu, or these three viruses together," Brindamour says. "It's frequent we find multiple viruses at the same time in kids - will that lead to more severe illness in kids or needing more mechanical ventilation and ICU admissions? We don't know yet."

Flu vaccines are currently being rolled out across Canada for everyone six months and older. Some provinces are prioritizing people at risk of flurelated complications, including hospital staff and patients, long-term care home staff and residents, pregnant people, seniors, children, Indigenous people and people with chronic health conditions.

Canada's National Advisory Council on Immunization ( $\mathrm{NACl}$ ) recommends that adults can get their flu and SARS-CoV-2 vaccines at the same time. Initially, $\mathrm{NACl}$ advised spacing out the vaccines by two weeks to monitor for adverse reactions, but that's no longer necessary for adults. Children will continue to receive the shots two weeks apart.
According to Bowdish, while influenza activity remains low, "I would definitely recommend that you get your COVID shot first because that's the real and pressing concern right now."

But even in a quiet influenza season, "every flu shot you get is a little immunological investment in the future," she says. "Sometimes, that strain might pop up again in decades from now and you'll still have that immunological memory."

\section{Diana Duong, CMAJ}

Content licence: This is an Open Access article distributed in accordance with the terms of the Creative Commons Attribution (CC BY-NC-ND 4.0) licence, which permits use, distribution and reproduction in any medium, provided that the original publication is properly cited, the use is noncommercial (i.e., research or educational use), and no modifications or adaptations are made. See: https://creativecommons.org/ licenses/by-nc-nd/4.0/ 